Statistical Methods in Medical Research

\title{
Inferential tools in penalized logistic regression for small and sparse data: A comparative study
}

\author{
Marianna Siino, Salvatore Fasola and Vito MR Muggeo
}

\begin{abstract}
This paper focuses on inferential tools in the logistic regression model fitted by the Firth penalized likelihood. In this context, the Likelihood Ratio statistic is often reported to be the preferred choice as compared to the 'traditional' Wald statistic. In this work, we consider and discuss a wider range of test statistics, including the robust Wald, the Score, and the recently proposed Gradient statistic. We compare all these asymptotically equivalent statistics in terms of interval estimation and hypothesis testing via simulation experiments and analyses of two real datasets. We find out that the Likelihood Ratio statistic does not appear the best inferential device in the Firth penalized logistic regression.
\end{abstract}

\section{Keywords}

Logistic regression, Firth penalized likelihood, sandwich formula, score statistic, gradient statistic

\section{Introduction}

Logistic regression is undoubtedly one of the most popular statistical models routinely used in many areas of applied statistics, such as Biology, Ecology and especially Medicine. ${ }^{1}$ Given a set of predictors, the model aims to assess the specific effect of one or more explanatory variables in the regression equation while providing estimates of the probability of the dichotomous outcome; excellent books describe it in detail. ${ }^{2,3}$

While standard logistic regression belongs to the main frame of statistical methods for applied research, its application in the presence of sparse data needs some attention and caution. ${ }^{4}$ Sparseness is sometimes referred as 'separation' of data, as described by Albert and Anderson, ${ }^{5}$ and it can be caused by occurrence of small sample size, and/or rare events, and/or unbalanced or highly predictive risk factors. Performance of the usual asymptotic procedures, point estimates, confidence intervals and hypothesis testing deteriorate in finite samples with sparseness of data. ${ }^{5,6}$ More specifically, point estimates are not guaranteed to exist, and when obtained, they can suffer from important bias leading to inconsistent estimators; interval estimates and $p$-values are doubtful due to the non-Normal sampling distribution of the usual test statistics.

Different strategies have been discussed in the literature, both 'corrective', and 'preventive'. The former approach means that the maximum likelihood estimates (MLE) are adjusted ex-post, ${ }^{7-11}$ whereas in the preventive approach the estimates solve modified estimating equations accounting for the bias of the MLEs. In the Firth's penalized approach, ${ }^{12}$ proper estimating equations are defined and parameter estimates are obtained accordingly, leading to estimators that are unbiased to the order $n^{-1}$. Such approach turns out particularly useful with nearly separated data: besides removing the first-order bias, the approach guarantees the point estimates to be finite even in the presence of monotone likelihood ${ }^{13}$ when the usual ML estimates do not exist. In the context of small samples and/or sparse data, some comparisons have been discussed ${ }^{14,15}$ ending up with the Firth penalized framework as the final recommendation. The Firth approach in logistic regression has been extended to multinomial responses ${ }^{16}$ and ordinal responses via cumulative link regression models; ${ }^{17}$ more generally, the penalized approach has also emerged noteworthy in developing prediction models. ${ }^{18}$ 
Within the Firth penalized logistic regression, several authors have discussed inferential tools to carry out inference on the model parameters. ${ }^{13,19}$ Comparisons have concerned the Likelihood Ratio and the Wald statistics only, the latter using a covariance matrix for estimators valid only in very large samples. In the end, likelihood-based confidence intervals are generally recommended over the Wald ones according to simulation evidences.

In this work, we focus on both interval estimation and hypothesis testing in the Firth penalized logistic regression. We extend discussion and comparisons involving also the other asymptotically equivalent test statistics: the Score statistic that, curiously, has been neglected in the aforementioned literature, and the recent Gradient statistic. ${ }^{20,21} \mathrm{We}$ also argue about inappropriateness of the usual Wald statistic that is usually considered in the literature.

The remainder of this paper is organized as follows. Section 2 summarizes the different test statistics employed to make inference, namely to get confidence intervals and $p$-values; Section 3 presents the design and the results of the simulation studies and Section 4 deals with the analysis of two well-known datasets in literature. The last Section is devoted to conclusions and final discussion.

\section{The four likelihood-based statistics}

Let $Y_{i}$ be the binary outcome variable for unit $i$, and $\boldsymbol{x}_{i}$ the $K$-dimensional covariate vector, possibly including exposures, confounders, predictors, and the 1 for model intercept. Interest lies on the conditional expected value $\pi_{i}=E\left[Y \mid \boldsymbol{x}_{i}\right]$ related to covariates via the logistic regression equation

$$
\operatorname{logit}\left(\pi_{i}\right)=\boldsymbol{x}_{\boldsymbol{i}}^{T} \boldsymbol{\beta}=\sum_{j=1}^{K} x_{i j} \beta_{j} .
$$

Maximum likelihood estimates (MLEs) of the regression parameters are usually obtained by equating to zero the score vector $\frac{\partial \ell(\boldsymbol{\beta})}{\partial \beta_{i}}=U_{j}(\boldsymbol{\beta}), j=0,1,2, \ldots, K$. As discussed in the previous section, sparseness poses serious issues in obtaining reliable point estimates and related quantities, and better estimating equations are obtained within the Firth penalized approach. Firth ${ }^{12,22}$ suggests the following modification of the classical score function $U_{j}(\boldsymbol{\beta})$ that allows to remove the $O\left(n^{-1}\right)$ bias of the ML estimates

$$
U_{j}^{*}(\boldsymbol{\beta})=U_{j}(\boldsymbol{\beta})+\frac{1}{2} \operatorname{trace}\left\{\boldsymbol{I}(\boldsymbol{\beta})^{-1} \frac{\partial \boldsymbol{I}(\boldsymbol{\beta})}{\partial \beta_{j}}\right\}, \quad j=0,1,2, \ldots, K
$$

where $\boldsymbol{I}(\boldsymbol{\beta})$ is the expected Fisher information. This is equivalent to use the penalized log-likelihood

$$
\ell^{*}(\boldsymbol{\beta})=\ell(\boldsymbol{\beta})+\log |\boldsymbol{I}(\boldsymbol{\beta})|^{\frac{1}{2}},
$$

where the penalty $|\boldsymbol{I}(\boldsymbol{\beta})|^{\frac{1}{2}}$ is the so-called Jeffrey's invariant prior in a Bayesian context. ${ }^{4,12,23,24}$

In the following sections, we summarize some statistics on which inference can be based: these are all likelihood-based, and refer to the same standard normal null distribution for large samples. However, in small to moderate samples, they can perform quite differently, as discussed later. We will indicate the full penalized ML estimate via $\hat{\boldsymbol{\beta}}^{*}$, and the restricted one via $\hat{\boldsymbol{\beta}}_{\mathbf{0}}^{*}$; namely $\hat{\boldsymbol{\beta}}_{\mathbf{0}}^{*}$ is the ML estimate of $\boldsymbol{\beta}$ given $\beta_{j}=\beta_{0 j}$ fixed under the null hypothesis.

\section{I The Likelihood Ratio statistic}

The Penalized Likelihood Ratio statistic is defined as the usual Likelihood Ratio statistic but involving the penalized log-likelihood (equation (3)). Thus, it is

$$
L=\operatorname{sign}\left(\hat{\beta}_{j}^{*}-\beta_{0 j}\right) \sqrt{-2\left\{\ell^{*}\left(\hat{\boldsymbol{\beta}}^{*}\right)-\ell^{*}\left(\hat{\boldsymbol{\beta}}_{0}^{*}\right)\right\}} .
$$

Several authors have claimed that the penalized Likelihood Ratio statistic is the preferred choice when using the Firth penalty in logistic regression ${ }^{13,14,19}$ 


\subsection{The Wald statistic}

The Wald statistic for the $j$ th regression parameter is naturally defined as

$$
W=\frac{\hat{\beta}_{j}^{*}-\beta_{0 j}}{\operatorname{var}\left(\hat{\beta}_{j}^{*}\right)^{1 / 2}}
$$

The key point here is how to compute $\operatorname{var}\left(\hat{\beta}_{j}^{*}\right)$, the $j$ th element on the main diagonal of the full covariance matrix $\boldsymbol{V}\left(\hat{\boldsymbol{\beta}}^{*}\right)$. All the aforementioned papers in literature use the inverse of the Information, namely $\boldsymbol{V}\left(\hat{\boldsymbol{\beta}}^{*}\right) \approx \boldsymbol{I}^{-1}\left(\hat{\boldsymbol{\beta}}^{*}\right)$. However, it should be acknowledged the $\boldsymbol{I}^{-1}$ is not the right approach to compute the variance of the estimator, at least in moderate samples. In fact, from basics of statistical inference, the asymptotic variance of the ML estimator comes from the linear expansion of the score, leading to the so-called sandwich formula, ${ }^{25}$ which reduces to $\boldsymbol{I}^{-1}$ only if the second Bartlett identity holds. In the Firth penalized framework, the simple approximation $\boldsymbol{V}\left(\hat{\boldsymbol{\beta}}^{*}\right) \approx \boldsymbol{I}^{-1}\left(\hat{\boldsymbol{\beta}}^{*}\right)$ holds only in very large samples when the penalty effect gets negligible. In particular, in small to moderate samples, $\boldsymbol{I}^{-1}\left(\hat{\boldsymbol{\beta}}^{*}\right)$ overestimates the true estimates uncertainty. This crucial issue appears to have been neglected in literature, ${ }^{13,16,19}$ causing a (pointless) 'bad reputation' of the Wald statistic. Hence, a more reliable asymptotic variance for $\hat{\boldsymbol{\beta}}^{*}$ is provided by the sandwich formula $\boldsymbol{H}^{*}\left(\hat{\boldsymbol{\beta}}^{*}\right)^{-1} \boldsymbol{I}\left(\hat{\boldsymbol{\beta}}^{*}\right) \boldsymbol{H}^{*}\left(\hat{\boldsymbol{\beta}}^{*}\right)^{-1}$. It should be noted that any adjective 'unpenalized' or 'penalized' (and corresponding asterisk) to be placed before 'Information' is meaningless. There is a unique Information, hereafter denoted by $\boldsymbol{I}=\operatorname{var}(\boldsymbol{U})=\operatorname{var}\left(\boldsymbol{U}^{*}\right)$; in fact the penalized and unpenalized score have the same variance since penalty in equation (2) does not depend on data. Evaluating the hessian $\boldsymbol{H}^{*}(\boldsymbol{\beta})$ requires the second derivatives of the penalized log-likelihood; even if these could be obtained analytically or numerically, via finite differences for instance, we found out that computing the hessian using the idea of induced smoothing ${ }^{26}$ works better in practice.

We use $W_{S}$ to indicate the 'fair' Wald statistic using the sandwich variance, and $W$ to mean the 'unfair' one using the simple $\boldsymbol{I}^{-1}$.

\subsection{The Score statistic}

The Score statistic is well known in the mainstream inference background, but its spread in applications is somewhat limited with respect to the Wald and Likelihood Ratio statistics. Such limited diffusion reflects in the Firth penalized logistic regression framework, where it appears to have been not yet discussed.

The penalized Score takes the form

$$
S=U_{j}^{*}\left(\hat{\boldsymbol{\beta}}_{0}^{*}\right) \sqrt{\boldsymbol{I}^{-1}\left(\hat{\boldsymbol{\beta}}_{0}^{*}\right)_{j j}}
$$

where $\boldsymbol{I}^{-1}\left(\hat{\boldsymbol{\beta}}_{0}^{*}\right)_{j j}$ is the $j$ th element on the main diagonal of the inverse of the variance of the conditional Score $U_{j}^{*}$. It should be noted that $\boldsymbol{U}^{*}$ is biased, namely its expected value is not zero due to the penalty in equation (2); however, such bias appears to have a negligible effect on the limit distribution of $S$, making the standard Normal a valid reference distribution to carry out inference on the regression parameters; simulation studies later bear this out. Moreover, as discussed elsewhere ${ }^{27}$ the Jeffrey prior (and the Firth penalty accordingly) is data dependent: this means that it would not be possible to discuss a general behaviour of the bias of the score.

\subsection{The Gradient statistic}

The Gradient statistic ${ }^{20}$ is a relatively new statistic introduced as a possibly simpler alternative to the most popular Likelihood Ratio, Wald and Score statistics. ${ }^{21}$ The Gradient statistic can be derived through the geometric mean between $W$ and $S$, namely

$$
G=\operatorname{sign}\left(\widehat{\beta}_{j}^{*}-\beta_{0 j}\right) \sqrt{\left(\widehat{\beta}_{j}^{*}-\beta_{0 j}\right) U_{j}^{*}\left(\hat{\boldsymbol{\beta}}_{0}^{*}\right)}
$$


However, due to lack of the second Bartlett identity, $G$ may be affected by the same inaccuracies of $W$. Thus, a robust formulation is

$$
G_{S}=\operatorname{sign}\left(\hat{\beta}_{j}^{*}-\beta_{0 j}\right) \sqrt{\left(\hat{\beta}_{j}^{*}-\beta_{0 j}\right) U_{j}^{*}\left(\hat{\boldsymbol{\beta}}_{\mathbf{0}}^{*}\right)\left[\boldsymbol{H}^{*}\left(\hat{\boldsymbol{\beta}}^{*}\right) \boldsymbol{I}^{-1}\left(\hat{\boldsymbol{\beta}}^{*}\right)\right]_{j j}}
$$

which comes from a multidimensional characterization of the geometric mean between $W_{S}$ and $S .^{28}$

\section{Simulation study}

We carry out a simulation study to assess the properties of the aforementioned statistics in making inference on the regression parameters in Firth's penalized logistic regression: the penalized LR (equation (4)), the Wald statistic (equation (5)) and its robust counterpart $W_{S}$ using the sandwich variance, the Score statistic (equation (7)), and the Gradient statistics (equations (7) and (8)).

We consider different scenarios to induce, to some extend, sparseness in the data: small to moderate sample sizes and highly predictive risk factors. Also both categorical and numerical covariates are considered. To provide recommendations with practical interest, we consider scenarios with one covariate only (and very small sample $n=20)$, and also three covariates as detailed below.

For each scenario, we generate $B=5000$ samples of Bernoulli data $Y_{i} \sim \operatorname{Ber}\left(\pi_{i}\right)$, where $\operatorname{logit}\left(\pi_{i}\right)=\eta_{i}$. In the single-covariate case, we consider three different sample sizes $n \in\{20,50,100\}$, and $\eta_{i}=1+\beta x_{i}$ where the covariate is continuous or binary. To define the covariate, we extract values $z_{i} \sim \mathcal{N}(0,1)$, and we simply set $x_{i}=z_{i}$ to get a continuous covariate, or $x_{i}=I\left(z_{i}>0\right)$ to obtain a binary covariate.

In the multiple-covariate case, $\eta_{i}=1+\beta x_{1 i}+0.5 x_{2 i}-0.5 x_{3 i}$, where to define the covariates, correlated multinormal values are drawn

$$
\left[\begin{array}{l}
z_{1 i} \\
z_{2 i} \\
z_{3 i}
\end{array}\right] \sim \mathcal{N}\left(\left[\begin{array}{l}
0 \\
0 \\
0
\end{array}\right],\left[\begin{array}{ccc}
1 & 0.6 & -0.6 \\
0.6 & 1 & 0 \\
-0.6 & 0 & 1
\end{array}\right]\right)
$$

and $x_{2 i}=I\left(z_{2 i}>0\right)$ (binary covariate), $x_{3 i}=z_{3 i}$ (continuous covariate). The covariate of interest $x_{1}$ is obtained via $x_{1 i}=I\left(z_{1 i}>0\right)$ or $x_{1 i}=z_{1 i}$. Due to the presence of additional covariates, we focus only on medium to large sample sizes, namely $n \in\{50,100\}$.

We first address interval estimation and use the statistics to derive confidence intervals for the regression coefficient of interest: we assess the performance via coverage levels and average widths. Then, we use the statistics for hypothesis testing by assessing empirical type I error and power rates. In the simulation runs, we do not distinguish between separated or nearly separated datasets, since the Firth penalized approach always returns finite and reliable estimates in practice.

\section{I Interval estimation}

Let $T\left(\beta_{0 j}\right)$ be one of the pivot statistics discussed in Section 2 evaluated at the candidate value $\beta_{0 j}$. A $(1-\alpha) 100 \%$ confidence interval (CI) for the parameter of interest $\beta_{j}$ is defined as

$$
\mathrm{CI}=\left\{\beta_{0 j} \in \mathbb{R}: z_{\alpha / 2} \leq T\left(\beta_{0 j}\right) \leq z_{1-\alpha / 2}\right\}
$$

where $z_{\alpha / 2}$ and $z_{1-\alpha / 2}$ are the quantiles of the standard normal distribution for a given $\alpha$.

Simulated data are obtained as described above, with values for the interest parameter $\beta \in\{0.5,1.5\}$, both in single or multiple covariate context. For the $95 \%$ CI of the interest parameter, Tables 1 and 2 report the empirical coverage levels (CL), the average widths (AW $\left.=\sum_{b}\left\{\mathrm{Upp}_{b}-\mathrm{Low}_{b}\right\} / B\right)$, and the average symmetry ratios given by $\mathrm{AS}=\sum_{b}\left\{\left(\mathrm{Upp}_{b}-\hat{\beta}_{b}\right) /\left(\hat{\beta}_{b}-\mathrm{Low}_{b}\right)\right\} / B$, where in each replicate $b, \hat{\beta}_{b}$ is the estimate, and $\mathrm{Low}_{b}$ and $\mathrm{Upp}_{b}$ are the lower and upper confidence limits. This ratio will be one for symmetric CI (such as those returned by $W$ and $W_{S}$ ) and will get more different from one as the asymmetry of the CI increases.

Despite that the Likelihood Ratio statistic performs rather fairly, it does not appear to be 'the best' inferential device in the Firth penalized logistic regression, as claimed in previous works. In general, the CI performances, in terms of coverage level, are similar using the other statistics with some light differences. The 'usual' Wald test 
Table I. Empirical coverage levels, average widths, and average symmetry ratios (based on 5000 runs) of $95 \%$ confidence intervals for $\beta_{1}$ according to the different statistics: Wald $(W)$, Robust-Wald $\left(W_{S}\right)$, Likelihood Ratio $(L)$, Score $(S)$, Gradient $(G)$ and RobustGradient $\left(G_{S}\right)$.

\begin{tabular}{|c|c|c|c|c|c|c|c|c|c|c|c|c|c|}
\hline \multirow[b]{3}{*}{$n$} & \multirow[b]{3}{*}{ stats } & \multicolumn{6}{|c|}{ Binary covariate } & \multicolumn{6}{|c|}{ Normal covariate } \\
\hline & & \multicolumn{3}{|c|}{$\beta=0.5$} & \multicolumn{3}{|c|}{$\beta=1.5$} & \multicolumn{3}{|l|}{$\beta=0.5$} & \multicolumn{3}{|c|}{$\beta=\mathrm{I} .5$} \\
\hline & & $\mathrm{CL}$ & AW & AS & $\mathrm{CL}$ & AW & AS & $\mathrm{CL}$ & AW & AS & $\mathrm{CL}$ & AW & AS \\
\hline \multirow[t]{6}{*}{20} & W & 0.990 & 4.75 & 1.00 & 0.984 & 5.60 & 1.00 & 0.988 & 2.44 & 1.00 & 0.951 & 3.30 & 1.00 \\
\hline & $W_{S}$ & 0.954 & 4.13 & 1.00 & 0.962 & 4.49 & 1.00 & 0.962 & 2.21 & 1.00 & 0.901 & 2.64 & 1.00 \\
\hline & $L$ & 0.957 & 4.91 & 1.14 & 0.976 & 6.10 & $\mathrm{I} .43$ & 0.964 & 2.52 & 1.23 & 0.961 & 3.62 & 1.64 \\
\hline & $S$ & 0.953 & 3.85 & 0.99 & 0.955 & 4.33 & 0.97 & 0.954 & 2.19 & 1.10 & 0.929 & 3.21 & 1.29 \\
\hline & $G$ & 0.948 & 5.63 & 1.33 & 0.975 & 7.57 & 1.96 & 0.954 & 2.74 & 1.40 & 0.958 & 4.04 & 2.14 \\
\hline & $G_{S}$ & 0.934 & 5.00 & 1.25 & 0.967 & 6.02 & 1.72 & 0.943 & 2.60 & 1.38 & 0.933 & 3.62 & 2.04 \\
\hline \multirow[t]{6}{*}{50} & $W$ & 0.968 & 2.83 & 1.00 & 0.973 & 3.71 & 1.00 & 0.972 & $1.4 \mathrm{I}$ & 1.00 & 0.949 & 1.99 & 1.00 \\
\hline & $W_{S}$ & 0.953 & 2.68 & 1.00 & 0.952 & 3.18 & 1.00 & 0.961 & 1.36 & 1.00 & 0.927 & 1.81 & 1.00 \\
\hline & $L$ & 0.951 & 2.86 & 1.07 & 0.963 & 3.94 & 1.36 & 0.954 & $1.4 \mathrm{I}$ & 1.13 & 0.948 & 2.05 & $\mathrm{I} .34$ \\
\hline & $S$ & 0.950 & 2.60 & 1.00 & 0.957 & 3.23 & 0.98 & 0.953 & 1.31 & 1.03 & 0.940 & 1.95 & 1.07 \\
\hline & $G$ & 0.946 & 2.96 & 1.13 & 0.960 & 4.42 & 1.68 & 0.951 & 1.44 & 1.21 & 0.947 & 2.13 & 1.55 \\
\hline & $G_{S}$ & 0.942 & 2.85 & I.II & 0.930 & 3.86 & 1.58 & 0.947 & 1.42 & 1.20 & 0.937 & 2.03 & 1.52 \\
\hline \multirow[t]{6}{*}{100} & $W$ & 0.953 & 1.95 & 1.00 & 0.97 I & 2.56 & 1.00 & 0.962 & 0.96 & 1.00 & 0.954 & 1.37 & 1.00 \\
\hline & $W_{S}$ & 0.948 & 1.91 & 1.00 & 0.946 & 2.35 & 1.00 & 0.958 & 0.95 & 1.00 & 0.946 & 1.31 & 1.00 \\
\hline & $L$ & 0.946 & 1.95 & 1.04 & 0.957 & 2.63 & 1.22 & 0.955 & 0.96 & 1.09 & 0.954 & 1.39 & 1.22 \\
\hline & $S$ & 0.946 & 1.87 & 1.00 & 0.954 & 2.38 & 0.99 & 0.952 & 0.93 & 1.01 & 0.951 & 1.35 & 1.03 \\
\hline & $G$ & 0.943 & 1.98 & 1.07 & 0.952 & 2.76 & 1.39 & 0.952 & 0.97 & 1.13 & 0.954 & 1.41 & 1.35 \\
\hline & $G_{S}$ & 0.940 & 1.95 & 1.07 & 0.940 & 2.59 & 1.36 & 0.951 & 0.97 & 1.13 & 0.949 & 1.38 & 1.34 \\
\hline
\end{tabular}

Note: Boldfaces refer to $C L$ outside the 'plausible' range $.95 \pm 2.57\{(.95 \cdot .05) / B\}^{1 / 2}$. The logistic regression equation is logit $\left(\pi_{i}\right)=1+\beta x_{i}($ see text for details).

Table 2. Empirical coverage levels, average widths, and average symmetry ratios (based on 5000 runs) of the $95 \%$ confidence intervals for $\beta_{1}$ according to the different statistics: Wald $(W)$, Robust-Wald $\left(W_{S}\right)$, Likelihood Ratio $(L)$, Score $(S)$, Gradient $(G)$ and Robust-Gradient $\left(G_{S}\right)$.

\begin{tabular}{|c|c|c|c|c|c|c|c|c|c|c|c|c|c|}
\hline \multirow[b]{3}{*}{$n$} & \multirow[b]{3}{*}{ stats } & \multicolumn{6}{|c|}{ Binary covariate } & \multicolumn{6}{|c|}{ Normal covariate } \\
\hline & & \multicolumn{3}{|c|}{$\beta=0.5$} & \multicolumn{3}{|c|}{$\beta=\mathrm{I} .5$} & \multicolumn{3}{|c|}{$\beta=0.5$} & \multicolumn{3}{|c|}{$\beta=\mathrm{I} .5$} \\
\hline & & $\mathrm{CL}$ & AW & AS & $\mathrm{CL}$ & AW & AS & $\mathrm{CL}$ & AW & AS & $\mathrm{CL}$ & AW & AS \\
\hline \multirow[t]{6}{*}{50} & W & 0.974 & 4.10 & 1.00 & 0.969 & 4.93 & 1.00 & 0.969 & 2.40 & 1.00 & 0.962 & 3.03 & 1.00 \\
\hline & $W_{S}$ & 0.952 & 3.82 & 1.00 & 0.955 & 4.29 & 1.00 & 0.955 & 2.30 & 1.00 & 0.940 & 2.76 & 1.00 \\
\hline & $L$ & 0.952 & 4.23 & 1.10 & 0.966 & 5.50 & 1.37 & 0.950 & 2.42 & 1.10 & 0.951 & 3.16 & 1.30 \\
\hline & $S$ & 0.954 & 3.78 & 1.00 & 0.959 & 4.51 & 1.00 & 0.949 & 2.24 & 1.03 & 0.950 & 2.96 & 1.10 \\
\hline & $G$ & 0.946 & 4.50 & 1.19 & 0.964 & 6.34 & 1.76 & 0.943 & 2.50 & 1.16 & 0.947 & 3.31 & 1.49 \\
\hline & $G_{s}$ & 0.932 & 4.22 & 1.16 & 0.951 & 5.40 & 1.62 & 0.936 & 2.43 & 1.15 & 0.932 & 3.10 & 1.46 \\
\hline \multirow[t]{6}{*}{100} & W & 0.963 & 2.75 & 1.00 & 0.967 & 3.52 & 1.00 & 0.960 & 1.62 & 1.00 & 0.956 & 2.02 & 1.00 \\
\hline & $W_{s}$ & 0.956 & 2.66 & 1.00 & 0.945 & 3.17 & 1.00 & 0.951 & 1.59 & 1.00 & 0.948 & 1.93 & 1.00 \\
\hline & $L$ & 0.955 & 2.77 & 1.05 & 0.957 & 3.77 & 1.27 & 0.949 & 1.62 & 1.06 & 0.952 & 2.04 & 1.18 \\
\hline & $S$ & 0.953 & 2.64 & 1.00 & 0.957 & 3.31 & 1.00 & 0.949 & 1.55 & 1.01 & 0.952 & 1.98 & 1.03 \\
\hline & $G$ & 0.952 & 2.83 & 1.09 & 0.953 & 4.09 & 1.51 & 0.946 & 1.64 & 1.10 & 0.951 & 2.08 & 1.29 \\
\hline & $G_{S}$ & 0.944 & 2.75 & 1.08 & 0.931 & 3.68 & $\mathrm{I} .44$ & 0.943 & 1.62 & 1.10 & 0.945 & 2.02 & 1.28 \\
\hline
\end{tabular}

Note: Boldfaces refer to $C L$ outside the 'plausible' range $.95 \pm 2.57\{(.95 \cdot .05) / B\}^{1 / 2}$. The logistic regression equation is $\operatorname{logit}\left(\pi_{i}\right)=\mathrm{I}+\beta \mathrm{x}_{1 i}+0.5 x_{2 i}-0.5 x_{3 i}$ (see text for details). 
heavily overestimates the estimator variance and thus provides wider CIs with coverage levels higher than the nominal one, even if $n=100$. In the more difficult scenario with small samples and strong predictor (that is $n=20$ and $\beta=1.5), L$ exhibits a conservative behaviour with somewhat large average widths: even with binary covariate, the average with of $L$ is about $40 \%$ larger than the average width from $S$.

Overall, Score-based CIs appear to perform slightly better than the other competitors, even in the most difficult scenarios with small samples $(n=20)$ and strong predictors causing sparsity and sampling zeros. The coverage levels are quite close to the nominal $95 \%$ and the average width is always the lowest. The top performance of $S$ is likely due to its simple structure: it is a sum of random variables and thus it is expected to converge quite fastly to the Normal distribution, and moreover, as discussed in the previous section, its exact variance is represented by the classical Fisher information matrix; curiously, its bias appears to have a negligible effect. Interestingly, the robust Wald statistic attains pretty good coverage levels with comparable average widths, sometimes even better than the Likelihood Ratio statistic. The good performance of $W_{S}$ also gives evidence that the Normal distribution of the regression coefficients estimator is not an issue actually, as long as the Firth penalization is employed and a finite estimate is guaranteed. This result is coherent with discussion reported in Royall. ${ }^{25}$ The Gradient statistics provide CIs with acceptable coverage levels but somewhat wider average widths; in particular the simple $G$, which does not require computation of the second derivatives, produces CIs with the largest width. Also the asymmetry amount of CIs based on $G$ and $G_{S}$ is fairly larger than the others.

Results for multiple (correlated) covariates do not differ with respect to the one-covariate models, and therefore they will not be further discussed.

\subsection{Hypothesis testing}

All the statistics presented in Section 2 can be clearly used for hypothesis testing. Moreover, in addition to the six likelihood-based statistics, we also consider a permutation-based test based on the Likelihood Ratio statistic. ${ }^{29}$ Here the null distribution is obtained via 1000 permutations of residuals and the $p$-value is computed as the portion of permuted values exceeding the observed value in the sample. Note that such approach corresponds to the exact conditional logistic regression when there is a single covariate. Here we focus on testing for the effect of the interest covariate, namely the hypothesis $H_{0}: \beta=0$ versus $H_{1}: \beta \neq 0$. The power functions of all statistics under investigation are obtained at seven values of $\beta \in\{ \pm 1.5, \pm 1.0, \pm 0.5,0\}$. Tables 3 and 4 portray the empirical rejection rates at nominal level 0.05 for the single and multiple covariate cases, respectively. Clearly, the entries in the middle columns represent the size, i.e. the type I error probability.

As for interval estimation, results again suggest that the Likelihood Ratio statistic does not perform the best. Under the null hypothesis, all the considered test statistics exhibit acceptable empirical rejection rates. $W$ is affected by the wrong variance formula and thus it exhibits the lowest rejection rates especially in small to moderate samples. As expected, there is no uniformly most powerful test, and the behaviour of the test statistics depends on the true value of the regression coefficient. Both the Gradient statistics, and in particular the robust one $G_{S}$, are featured by high power, especially on the 'right side' when the parameter takes positive values; however, $G_{S}$ tends to return high rejection rates also under the null hypothesis, making it rather useless, at least in theory. In large samples, the penalty effect vanishes and, to some extent, differences disappear.

When the model involves multiple covariates, findings are substantially unchanged for the six likelihood-based statistics, but the permutational statistic exhibits comparatively better performance.

\section{Examples}

We illustrate the different statistics and relevant findings on two real datasets previously analysed in the literature.

\section{I Osteogenic sarcoma data}

The first dataset is taken from Metha and Patel. ${ }^{30}$ The data refer to a study on $n=46$ patients with osteogenic sarcoma. The three-year disease-free interval (DFI3) is the response variable, while the categorical explanatory variables are gender (SEX), the presence of any osteoid pathology (AOP) and lymphocytic infiltration (LI). The main interest lies on the effect of LI. The classical MLEs do not exist finite, because of separation caused by the variable LI: there are no disease-free individuals among subjects without infiltration. We fit a penalized logistic regression model with additive linear effects and compute the $95 \%$ confidence intervals for the regression coefficient of covariate LI; results are reported in Table 5. 
Table 3. Empirical rejection rates (based on 5000 runs) for hypothesis testing $H_{0}: \beta=0$ vs. $H_{1}: \beta \neq 0$ for different test statistics: Wald $(W)$, Robust-Wald $\left(W_{S}\right)$, Likelihood Ratio $(L)$, Permutation Likelihood Ratio $\left(L_{p}\right)$, Score $(S)$, Gradient $(G)$ and Robust-Gradient $\left(G_{S}\right)$.

\begin{tabular}{|c|c|c|c|c|c|c|c|c|}
\hline \multirow[b]{2}{*}{$n$} & \multirow[b]{2}{*}{ Test } & \multicolumn{7}{|c|}{ True $\beta$ values } \\
\hline & & -1.5 & -1.0 & -0.5 & 0 & 0.5 & 1.0 & 1.5 \\
\hline & & \multicolumn{7}{|c|}{ Binary covariate } \\
\hline \multirow[t]{7}{*}{20} & W & 0.208 & 0.084 & 0.021 & 0.009 & 0.013 & 0.014 & 0.028 \\
\hline & $W_{S}$ & 0.312 & 0.155 & 0.056 & 0.038 & 0.059 & 0.097 & 0.167 \\
\hline & $L$ & 0.339 & 0.176 & 0.063 & 0.039 & 0.047 & 0.076 & 0.122 \\
\hline & $L_{p}$ & 0.270 & 0.128 & 0.049 & 0.026 & 0.039 & 0.059 & 0.099 \\
\hline & $S$ & 0.361 & 0.194 & 0.074 & 0.044 & 0.054 & 0.083 & 0.127 \\
\hline & $G$ & 0.353 & 0.190 & 0.075 & 0.048 & 0.061 & 0.098 & 0.164 \\
\hline & $G_{S}$ & 0.354 & 0.193 & 0.078 & 0.059 & 0.102 & 0.165 & 0.273 \\
\hline \multirow[t]{7}{*}{50} & W & 0.669 & 0.351 & 0.103 & 0.034 & 0.074 & 0.163 & 0.272 \\
\hline & $W_{S}$ & 0.674 & 0.364 & 0.116 & 0.046 & 0.100 & 0.231 & 0.432 \\
\hline & $L$ & 0.701 & 0.390 & 0.128 & 0.050 & 0.102 & 0.231 & 0.429 \\
\hline & $L_{p}$ & 0.664 & 0.358 & 0.106 & 0.040 & 0.089 & 0.210 & 0.391 \\
\hline & $S$ & 0.725 & 0.410 & 0.132 & 0.051 & 0.105 & 0.234 & 0.424 \\
\hline & $G$ & 0.710 & 0.406 & 0.131 & 0.053 & 0.118 & 0.256 & 0.460 \\
\hline & $G_{S}$ & $0.7 \mid 4$ & 0.408 & 0.133 & 0.055 & 0.130 & 0.283 & 0.511 \\
\hline \multirow[t]{8}{*}{100} & W & 0.948 & 0.632 & 0.200 & 0.042 & 0.158 & 0.421 & 0.690 \\
\hline & $W_{S}$ & 0.948 & 0.636 & 0.204 & 0.045 & 0.168 & 0.455 & 0.733 \\
\hline & $L$ & 0.955 & 0.647 & 0.217 & 0.049 & 0.174 & 0.469 & 0.739 \\
\hline & $L_{p}$ & 0.941 & 0.615 & 0.199 & 0.043 & 0.160 & 0.437 & 0.716 \\
\hline & $S^{r}$ & 0.956 & 0.655 & 0.219 & 0.050 & 0.175 & 0.467 & 0.732 \\
\hline & $G$ & 0.955 & $0.65 I$ & 0.219 & 0.051 & 0.178 & 0.475 & 0.753 \\
\hline & $G_{S}$ & 0.955 & 0.651 & 0.220 & 0.053 & 0.187 & 0.491 & 0.771 \\
\hline & & \multicolumn{7}{|c|}{ Normal covariate } \\
\hline \multirow[t]{7}{*}{20} & W & 0.323 & 0.143 & 0.036 & 0.006 & 0.028 & 0.143 & 0.335 \\
\hline & $W_{S}$ & 0.587 & $0.34 I$ & 0.113 & 0.029 & 0.097 & 0.328 & 0.607 \\
\hline & $L$ & 0.653 & 0.392 & 0.133 & 0.040 & 0.125 & 0.370 & 0.665 \\
\hline & $L_{p}$ & 0.673 & 0.417 & 0.148 & 0.046 & 0.140 & 0.405 & 0.692 \\
\hline & $S$ & 0.666 & 0.417 & 0.144 & 0.046 & 0.131 & 0.392 & 0.673 \\
\hline & $G$ & 0.697 & 0.437 & 0.162 & 0.054 & 0.149 & 0.423 & 0.708 \\
\hline & $G_{S}$ & 0.732 & 0.478 & 0.183 & 0.066 & 0.170 & 0.456 & 0.735 \\
\hline \multirow[t]{7}{*}{50} & $W$ & 0.962 & 0.749 & 0.238 & 0.027 & 0.247 & 0.747 & $0.97 \mid$ \\
\hline & $W_{S}$ & 0.970 & 0.780 & 0.277 & 0.034 & 0.280 & 0.779 & 0.977 \\
\hline & $L$ & 0.973 & 0.812 & 0.313 & 0.043 & 0.313 & 0.807 & 0.981 \\
\hline & $L_{p}$ & 0.976 & 0.821 & 0.322 & 0.047 & 0.324 & 0.814 & 0.982 \\
\hline & $S$ & 0.975 & 0.820 & 0.324 & 0.043 & 0.318 & 0.812 & 0.983 \\
\hline & $G$ & 0.976 & 0.823 & 0.328 & 0.047 & 0.329 & 0.816 & 0.983 \\
\hline & $G_{S}$ & 0.978 & 0.834 & 0.339 & 0.049 & 0.340 & 0.823 & 0.984 \\
\hline \multirow[t]{7}{*}{100} & $w$ & 1.000 & 0.978 & 0.532 & 0.039 & 0.519 & 0.979 & 1.000 \\
\hline & $W_{S}$ & 1.000 & 0.980 & 0.546 & 0.043 & 0.530 & 0.981 & 1.000 \\
\hline & $L$ & 1.000 & 0.983 & 0.574 & 0.050 & 0.556 & 0.984 & 1.000 \\
\hline & $L_{p}$ & 1.000 & 0.983 & 0.575 & 0.051 & 0.561 & 0.984 & 1.000 \\
\hline & $S^{r}$ & 1.000 & 0.984 & 0.576 & 0.052 & 0.560 & 0.985 & 1.000 \\
\hline & $G$ & 1.000 & 0.985 & 0.581 & 0.052 & 0.565 & 0.985 & 1.000 \\
\hline & $G_{S}$ & 1.000 & 0.985 & 0.584 & 0.053 & 0.569 & 0.985 & 1.000 \\
\hline
\end{tabular}

Note: The nominal level is 0.05 and boldfaces refer to rates outside the 'plausible' range $.05 \pm 2.57\{(.95 \cdot .05) / B\}^{1 / 2}$. The logistic regression equation is $\operatorname{logit}\left(\pi_{i}\right)=\mathrm{I}+\beta x_{i}$ (see text for details). 
Table 4. Empirical rejection rates (based on 5000 runs) for hypothesis testing $H_{0}: \beta_{1}=0$ vs. $H_{1}: \beta_{1} \neq 0$ for different test statistics: Wald $(W)$, Robust-Wald $\left(W_{S}\right)$, Likelihood Ratio $(L)$, Permutation Likelihood Ratio $\left(L_{p}\right)$, Score $(S)$, Gradient $(G)$ and Robust-Gradient $\left(G_{S}\right)$.

\begin{tabular}{|c|c|c|c|c|c|c|c|c|}
\hline \multirow[b]{2}{*}{$n$} & \multirow[b]{2}{*}{ Test } & \multicolumn{7}{|c|}{ True $\beta$ values } \\
\hline & & -1.5 & -1 & -0.5 & 0 & 0.5 & I & 1.5 \\
\hline & & \multicolumn{7}{|c|}{ Binary covariate } \\
\hline \multirow[t]{7}{*}{50} & $W$ & 0.376 & 0.170 & 0.061 & 0.018 & 0.036 & 0.069 & 0.107 \\
\hline & $W_{S}$ & $0.41 \mathrm{I}$ & 0.199 & 0.075 & 0.036 & 0.077 & 0.148 & 0.227 \\
\hline & $L$ & 0.460 & 0.228 & 0.093 & 0.040 & 0.072 & 0.134 & 0.201 \\
\hline & $L_{p}$ & 0.468 & 0.234 & 0.099 & 0.043 & 0.083 & 0.162 & 0.245 \\
\hline & $S$ & 0.487 & 0.239 & 0.098 & 0.040 & 0.069 & 0.128 & 0.182 \\
\hline & $G$ & 0.477 & 0.236 & 0.100 & 0.046 & 0.085 & 0.151 & 0.225 \\
\hline & $G_{S}$ & 0.480 & 0.236 & 0.100 & 0.050 & 0.103 & 0.201 & 0.295 \\
\hline \multirow[t]{8}{*}{100} & $W$ & 0.730 & 0.368 & 0.123 & 0.041 & 0.089 & 0.207 & 0.347 \\
\hline & $W_{S}$ & 0.736 & 0.380 & 0.129 & 0.046 & 0.108 & 0.261 & 0.447 \\
\hline & $L$ & 0.763 & 0.410 & 0.140 & 0.052 & 0.109 & 0.255 & 0.434 \\
\hline & $L_{p}$ & 0.766 & 0.413 & 0.146 & 0.053 & 0.115 & 0.269 & 0.460 \\
\hline & $S$ & 0.773 & 0.421 & 0.144 & 0.052 & 0.107 & 0.243 & $0.4 \mathrm{II}$ \\
\hline & $G$ & 0.768 & 0.419 & 0.145 & 0.054 & 0.113 & 0.272 & 0.454 \\
\hline & $G_{S}$ & 0.768 & 0.418 & 0.145 & 0.056 & 0.129 & 0.307 & 0.503 \\
\hline & & \multicolumn{7}{|c|}{ Normal covariate } \\
\hline \multirow[t]{7}{*}{50} & $W$ & 0.962 & 0.749 & 0.238 & 0.027 & 0.247 & 0.747 & 0.971 \\
\hline & $W_{S}$ & 0.970 & 0.780 & 0.277 & 0.034 & 0.280 & 0.779 & 0.977 \\
\hline & $L$ & 0.973 & 0.812 & 0.313 & 0.043 & 0.313 & 0.807 & 0.981 \\
\hline & $L_{p}$ & 0.976 & 0.821 & 0.322 & 0.047 & 0.324 & 0.814 & 0.982 \\
\hline & $S$ & 0.975 & 0.820 & 0.324 & 0.043 & 0.318 & 0.812 & 0.983 \\
\hline & $G$ & 0.976 & 0.823 & 0.328 & 0.047 & 0.329 & 0.816 & 0.983 \\
\hline & $G_{S}$ & 0.978 & 0.834 & 0.339 & 0.049 & 0.340 & 0.823 & 0.984 \\
\hline \multirow[t]{7}{*}{100} & $W$ & 1.000 & 0.978 & 0.532 & 0.039 & 0.519 & 0.979 & 1.000 \\
\hline & $W_{S}$ & 1.000 & 0.980 & 0.546 & 0.043 & 0.530 & 0.981 & 1.000 \\
\hline & $L$ & 1.000 & 0.983 & 0.574 & 0.050 & 0.556 & 0.984 & 1.000 \\
\hline & $L_{p}$ & 1.000 & 0.983 & 0.575 & 0.051 & 0.561 & 0.984 & 1.000 \\
\hline & $S$ & 1.000 & 0.984 & 0.576 & 0.052 & 0.560 & 0.985 & 1.000 \\
\hline & $G$ & 1.000 & 0.985 & 0.581 & 0.052 & 0.565 & 0.985 & 1.000 \\
\hline & $G_{S}$ & 1.000 & 0.985 & 0.584 & 0.053 & 0.569 & 0.985 & 1.000 \\
\hline
\end{tabular}

Note: The nominal level is 0.05 and boldfaces refer to rates outside the 'plausible' range $.05 \pm 2.57\{(.95 \cdot .05) / B\}^{1 / 2}$. The logistic regression equation is $\operatorname{logit}\left(\pi_{i}\right)=\mathrm{I}+\beta \mathrm{x}_{1 i}+0.5 \mathrm{x}_{2 i}-0.5 \mathrm{x}_{3 i}$ (see text for details).

Table 5. Confidence intervals (95\%) (and relevant width) for $\beta_{L I}$ based on the Wald (W), Robust-Wald $\left(W_{S}\right)$, Likelihood Ratio $(L)$, Score $(S)$, Gradient $(G)$ and Robust-Gradient $\left(G_{S}\right)$ statistics.

\begin{tabular}{lcccccc}
\hline $\mathrm{Cl}$ & $W$ & $W_{S}$ & $L$ & $S$ & $G$ & $G_{S}$ \\
\hline Low & -5.504 & -4.637 & -7.363 & -4.804 & -10.148 & -10.161 \\
UpP & 0.582 & -0.286 & -0.188 & -0.104 & -0.356 & -0.355 \\
Width & 6.09 & 4.35 & 7.17 & 4.70 & 9.79 & 9.81 \\
\hline
\end{tabular}

The results are coherent with the previous simulation findings. The CIs based on the Gradient statistics are the widest and the most asymmetric, although they end up with a significant effect of LI. The CI coming from $W$ is also pretty wide but it is symmetric and includes the zero, leading to a (likely misleading) non-significant effect of the variable LI. On the other hand, the Score and the robust Wald CIs are the narrowest ones and also return a significant result for LI. 


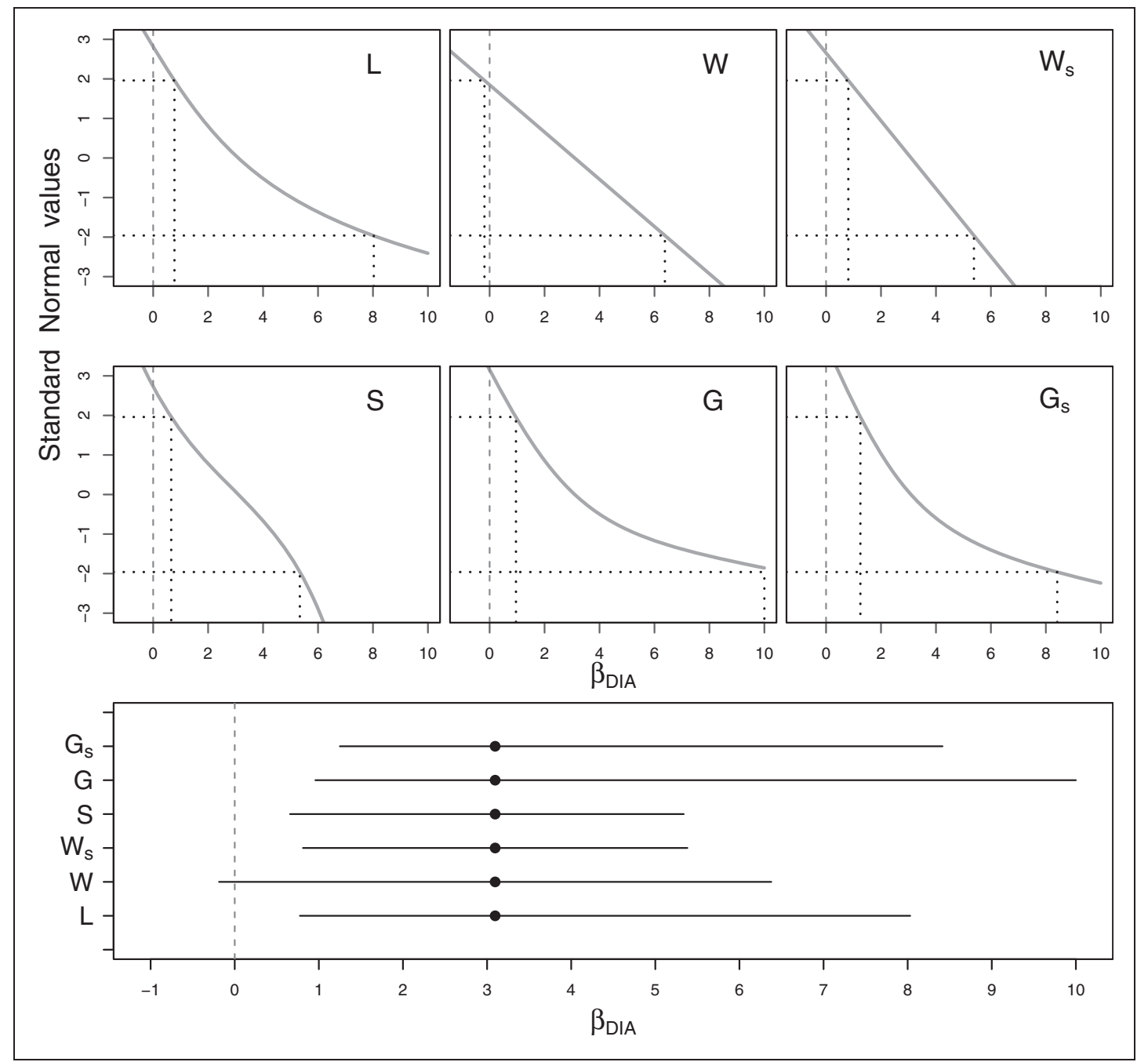

Figure I. The six likelihood-based statistics $\left(L=\right.$ Likelihood Ratio, $W=W a l d, W_{S}=$ robust Wald, $S=S c o r e, G=G r a d i e n t$, $G_{S}=$ robust Gradient) profiled and corresponding $95 \%$ confidence intervals for the parameter $\beta_{D I A}$ in the Urinary tract infection example.

\subsection{Urinary tract infection}

The second example concerns a retrospective case-control study on possible determinants of urinary infections on a sample of sexually active college women carried out at the University of Michigan. ${ }^{31,32}$

The 130 cases and 109 controls were classified according to six binary covariates, namely age (lower or greater than 23 years) and some indicators of sexual behaviour: use of oral contraceptive (OC), condom (VIC), lubricated condom (VICL), spermicide (VIS) or diaphragm (DIA). Since all the women using a diaphragm were cases, separation occurs and we estimate a penalized logistic regression model to obtain finite estimates. We focus on the effect of covariate 'using the diaphragm' (DIA) on the probability of infection, and compute the $95 \%$ confidence interval for corresponding regression coefficient $\beta_{D I A}$ using the different statistics. The intervals are represented in Figure 1, together with the six curves corresponding to the pivotal statistics $T\left(\beta_{D I A}\right)$ profiled. Again, the CIs based on $S$ and $W_{S}$ are the narrowest, while the CI based on $W$ leads to different, and possibly misleading, conclusions. Again the gradient statistics lead to quite large and asymmetric CI but not including the zero.

\section{Conclusions}

Small datasets are rather frequent in medical research, and it is therefore quite imperative from a statistical perspective to set up appropriate methods to obtain valid and reliable inferential procedures. At this aim, many 
authors have carried out comparisons among the different 'classical' options, but interest was limited to the 'usual' Wald and the Likelihood Ratio statistics. The usual Wald uses a simple but inappropriate formula for the estimates variance, i.e. the inverse of Information. However, as previously discussed, the inverse of Information is appropriate only in large samples. In small to moderate samples, when the penalty is not negligible, a sandwich formula turns out to be more appropriate, and the resulting robust Wald-based CIs have the correct coverage levels. Moreover, the satisfactory performance of the robust Wald suggests that Normality of the estimator is not actually an issue as claimed previously in the literature; instead, as discussed by Royall, ${ }^{25}$ using the appropriate variance appears to be enough to guarantee a large sample standard Normal distribution. Besides Wald and Likelihood Ratio, in this paper we have also discussed the Score, the Gradient statistics.

In the context of interval estimation, the Score-based approach appears to return CIs with correct coverage levels across the scenarios and regardless of the number of covariates in the model. For hypothesis testing problems, differences among the approaches are minor, with a noticeable good performance of the simple Gradient statistic; its ease of computation represents a noteworthy advantage, since it just needs the point estimate and the first derivative. From a practical viewpoint, the different statistics exhibit approximately the same computational burden: profiling is a necessary step to obtain the endpoints of the CI in all but one statistic: in fact the robust Wald statistic does not need profiling, but the (penalized) hessian has to be computed to obtain reliable standard errors via the sandwich formula. The permutational approach is the heaviest as the model has to be fitted at each permuted dataset.

For applications, currently the Firth procedure is implemented in most statistical softwares/languages: for instance, the brglm or pmlr packages in R, the firthlogit program in Stata, and the firth option to the model statement in proc logistic in SAS. Currently it seems that only the Likelihood Ratio or the 'unfair' Wald statistics have been implemented for confidence intervals or hypothesis testing; it is hoped that some of the 'alternative' statistics, such as the Score, are implemented to obtain results with better statistical properties. The supplementary material available online includes $\mathrm{R}$ code to compute Score-based CI and p-value (smm.sagepub.com).

Finally, application and comparisons of the aforementioned statistics in more general logistic regressions, such as the cumulative link models for ordinal responses, ${ }^{17}$ represent a noteworthy point to be investigated.

\section{Acknowledgement}

The authors would like to thank the reviewers for their insightful comments and suggestions which greatly improved the paper.

\section{Funding}

The author(s) received no financial support for the research, authorship, and/or publication of this article.

\section{References}

1. Steyerberg EW. Clinical prediction models: a practical approach to development, validation, and updating. New York, NY: Springer, 2008.

2. Harrell F. Regression modeling strategies. New York, NY: Springer, 2001.

3. Hosmerm D, Lemeshowm S and Sturdivant R. Applied logistic regression, 3rd ed. New York, NY: Wiley, 2013.

4. Hamra G, MacLehose R and Cole S. Sensitivity analyses for sparse-data problems using weakly informative bayesian priors. Epidemiology 2013; 24: 233-239.

5. Albert A and Anderson JA. On the existence of maximum likelihood estimates in logistic regression models. Biometrika 1984; 71: 1-10.

6. Jennings D. Judging inference adequacy in logistic regression. J Am Statis Assoc 1986; 81: 471-476.

7. Schaefer RL. Bias correction in maximum likelihood logistic regression. Stat Med 1983; 2: 71-78.

8. Cordeiro GM and McCullagh P. Bias correction in generalized linear models. J Royal Stat Soc Ser B (Methodological) 1991; 53: 629-643.

9. Bull SB, Greenwood CM and Hauck WW. Jackknife bias reduction for polychotomous logistic regression. Stat Med 1997; 16: $545-560$.

10. Cordeiro GM and Cribari-Neto F. On bias reduction in exponential and non-exponential family regression models. Commun Stat-Simul Computat 1998; 27: 485-500.

11. Leung DHY and Wang YG. Bias reduction using stochastic approximation. Australian \& New Zealand J Stat 1998; 40: 43-52.

12. Firth D. Bias reduction of maximum likelihood estimates. Biometrika 1993; 80: 27-38. 
13. Heinze G and Schemper M. A solution to the problem of separation in logistic regression. Stat Med 2002; 21: 2409-2419.

14. Heinze G. A comparative investigation of methods for logistic regression with separated or nearly separated data. Stat Med 2006; 25: 4216-4226.

15. Maiti T and Pradhan V. A comparative study of the bias corrected estimates in logistic regression. Stat Meth Med Res 2008; 17: 621-634.

16. Bull SB, Mak C and Greenwood CM. A modified score function estimator for multinomial logistic regression in small samples. Computat Stat Data Analysis 2002; 39: 57-74.

17. Kosmidis I. Improved estimation in cumulative link models. J Royal Stat Soc: Ser B (Statistical Methodology) 2014; 76: 169-196.

18. Moons K, Donders AR, Steyerberg E, et al. Penalized maximum likelihood estimation to directly adjust diagnostic and prognostic prediction models for overoptimism: a clinical example. J Clin Epidemiol 2004; 57: 1262-1270.

19. Bull SB, Lewinger JP and Lee SS. Confidence intervals for multinomial logistic regression in sparse data. Stat Med 2007; 26: $903-918$.

20. Terrell GR. The gradient statistic. Comput Sci Stat 2002; 34: 206-215.

21. Muggeo VMR and Lovison G. The 'three plus one' likelihood-based test statistics: unified geometrical and graphical interpretations. Am Statistician 2014; 68: 302-306.

22. Firth D. Generalized linear models and Jeffreys priors: an iterative weighted least-squares approach. In: Dodge Y and Whittaker J (eds) Computational statistics, vol 1. Neuchatel Switzerland, Springer, 1992, pp.553-557..

23. Jeffrey H. An invariant form for the prior probability in estimation problems. Proc Royal Soc London Series A, Math Phys Sci 1946; 186: 453-461.

24. Chen M, Ibrahim J and Kim S. Properties and implementation of Jeffreys's prior in binomial regression models. $J$ Am Stat Assoc 2008; 103: 1659-1664.

25. Royall RM. Model robust confidence intervals using maximum likelihood estimators. Int Stat Rev/Revue Internationale de Statistique 1986; 54: 221-226.

26. Jin Z, Shao Y and Ying Z. A monte carlo method for variance estimation for estimators based on induced smoothing. Biostatistics 2015; 16: 179-188.

27. Greenland S and Mansournia MA. Penalization, bias reduction, and default priors in logistic and related categorical and survival regressions. Stat Med 2015; 34: 3133-3143.

28. Lemonte AJ. On the gradient statistic under model misspecification. Stat Probabil Lett 2013; 83: 390-398.

29. Potter DM. A permutation test for inference in logistic regression with small- and moderate-sized data sets. Stat Med 2005; 24: 693-708.

30. Mehta CR and Patel NR. Exact logistic regression: theory and examples. Stat Med 1995; 14: $2143-2160$.

31. Foxman B, Marsh J, Gillespie B, et al. Condom use and first-time urinary tract infection. Epidemiology 1997; 8: 637-641.

32. Corcoran C, Mehta C, Patel N, et al. Computational tools for exact conditional logistic regression. Stat Med 2001; 20: 2723-2739. 